\title{
Abstracts
}

\section{Taking Up the Bet on Connections: a Municipal Contribution}

Globalisation, the buzzword of the late twentieth century, calls for the attention of historians. As others have already suggested, one way to contribute to the historicisation of the phenomena encapsulated in this idiom is to pay attention to connections over long periods. Connections between municipal governments and exchanges about the subject have both been neglected by historical scholarship for various reasons, but they can contribute to the history of the 'construction of the universal'. Indeed, the information systems of municipal connections - their vectors, actors and structures - have defined, intersected with, nourished or undergone a series of would-be universalist 'transboundary formations'. These formations are shifting combinations of values, collective actions, practices, rules, organisations and individuals, all of which are advanced as possible futures for mankind. By examining some of these discourses of 'social order' and 'world order' and the way in which they combine with municipal connections, this article attempts to produce the 'municipal contribution' announced in its title.

\section{The Science that Never Was: 'Communal Science' in France, I9I3-I949}

Attempts to develop a 'municipal science' bear witness to the challenges posed by the city to methods of government in the first decades of the twentieth century, particularly in France. Both in France and on the international scene, reformers sought new ways of thinking 'scientifically' about towns and their government. This article takes a serious look at 'municipal science', a fragile development which was never fully accepted in academic circles. It studies the endeavours to provide a scientific clothing for the reforming discourse developed by associations of town councillors and disseminated by teaching institutions or journals devoted to municipal administration. It also examines the contribution made by this crusade - at once political and scientific - to the renewal of public action through local authorities.

\section{The Union of Polish Cities in the Second Polish Republic, I9I8-I939: Discourses of Local Government in a Divided Land}

The new Polish state was founded more than Ioo years after Poland's partition by Prussia, Russia and Austria. The partitioned Polish lands had been included one way or another in the administrative structure of the ocupying powers, and the individuals who became active in urban issues in the new state were socialised by associations established by the partitioners. Poland became not only a arena for a meeting of Prussian, Russian and Austrian imaginations 
about local government but also a place with a great variety of municipal praxises as well. The author analyses different meanings of local government with special attention to those employed by municipal officers from Warsaw and Cracow within the Union of Polish Cities. There were strong regional cleavages in the Union, but the political development of the Polish state strengthened centralisation and the Union itself remained united.

\section{European Municipalism in the First Half of the Twentieth Century: the Socialist Network}

This article analyses the contribution of European socialism to the building of a variegated network of reformers in municipal politics from the end of the nineteenth century to the I950s. Unsuccessful as a network inside the Second International, a broader international federation of cities, the Union Internationale des Villes/International Union of Local Authorities (UIV/IULA), was proposed in I9I3. Belgian, French, Dutch and English socialist leaders remained strongly influential in this federation between the two world wars, working in connection with co-operative movements and the International Labour Office based in Geneva. The fifty years of debates and projects animated by the international journal Les Annales de la Régie Directe founded by the French socialist Edgard Milhaud allows us to follow the development of a generation of local reformers from the beginnings of municipalist thought and praxis up to the idea of building a decentralised European Community of cities and regional authorities.

\section{Cities against States? Hopes, Dreams and Shortcomings of the European Municipal Movement, I900-I960}

At the beginning of the twentieth century the growth of towns was accompanied by the birth of a European and international municipal movement. In I9I 3 the Union Internationale des Villes/International Union of Local Authorities (UIV/IULA) was founded in Ghent. This combined two approaches, one political and utopian, the other technical and professional. The former focused on turning cities into promoters of a project for cooperation at European and worldwide level; the latter on solving the problems caused by urbanisation. They shared a determination to pursue their objectives regardless of the barriers of culture and national boundaries, and of the administrative hierarchy between local and national government. Between the two world wars the UIV/IULA gradually lost its original utopian inspiration, which was taken up by the Conseil des Communes d'Europe/Council of European Municipalities, founded in Geneva in I95I with the aim of using the municipalities as a starting point to promote a federation of European states.

\section{Europe from the Bottom Up: Town Twinning in France during the Cold War}

Town twinning can be seen as the first activity to involve municipal institutions as such in wide-ranging and long-term international action. Twinning fits Eric Hobsbawm's definition of an 'invented tradition'. The French federalists played a decisive part in this process of invention, bringing European municipalities together in order to ensure an eventual European political union. Before long, however, the so-called bilingualist movement also turned to twinning as a way to further a universal mutual understanding that transcended the East-West divide. This resulted in a big clash between the French twinning organisations. Thus, the invention of town twinning needs to be studied alongside the dynamic political tensions that emerged on the French political scene during the Cold War. 


\section{Extraits}

\section{Le pari des connexions: une contribution municipale}

La 'mondialisation', thème valise de la fin du 2oème siècle, appelle de plus en plus l'attention des historiens. Certains ont déjà suggéré qu'une des manières de contribuer à historiciser les phénomènes emballés dans cette notion attrape-tout consistait à travailler le thème des connexions sur le long terme. Les connexions qui se sont dévéloppées entre les municipalités, et les échanges qui eurent lieu au sujet du gouvernement municipal, n'ont guère suscité le regard historien jusqu'à présent, mais leur étude peut contribuer à l'histoire de la 'construction de l'universel'. En effet, les systèmes d'information développés autour des connexions municipales - leurs vecteurs, leurs structures, leurs acteurs - ont défini, nourris, supportés ou rencontrés toute une série de 'formations transnationales'. Ces formations sont des combinaisons fluctuantes de valeurs, d'actions collectives, de pratiques, de règles, d'organisations et d'individus, qui se présentent comme des futurs possibles pour l'humanité. Le 'municipalisme' et les connexions municipales se combinent avec ces discours de l'ordre social et de l'ordre mondial, et l'étude de cet objet apparemment local contribue ainsi à notre compréhension du global.

\section{Une science non consolidée: la 'science communale' en France I9I3-I949}

Les tentatives de développer une 'science communale' témoignent des défis que la ville a représenté pour les méthodes de gouvernement dans les premières décennies du vingtième siècle. Tant en France que sur la scène internationale, des groupements réformateurs ont recherché des manières de penser 'scientifiquement' les villes et leur gouvernement. Cet article s'attache à retracer l'histoire de la 'science municipale' ou 'science communale' en France, tentative fragile qui ne trouva jamais sa place dans les structures de l'université française. Il étudie en particulier l'appareillage scientifique mis en place dans le discours réformateur par les diverses associations de municipalités et d'élus municipaux, et diffusé par des périodiques ou des lieux d'enseignement spécialisés dans l'administration municipale. C'est ce qui lui permet d'examiner les contributions de cette croisade scientifique et politique au renouvellement du mode d'action public des municipalités.

\section{L’Union des Villes Polonaises sous la Seconde République (I9I8-I939): les discours du gouvernement municipal dans un pays fragmenté}

Le nouvel état polonais fut créé plus de cent ans après la partition de la Pologne entre la Prusse, l'Autriche et la Russie. Les régions ainsi divisées prirent place dans les structures administratives des puissances occupantes, et les individus qui participèrent aux discussions sur les questions urbaines et municipales dans le nouvel état avaient été socialisés dans les contextes législatifs et les milieux associatifs de ces mêmes puissances. Le nouvel état polonais fut en conséquence à la fois un lieu de rencontre des définitions prussiennes, russes ou autrichiennes du gouvernement municipal, mais aussi un pays marqué par une grande variété de pratiques du gouvernement municipal. L'auteur analyse ici ces différentes significations du gouvernement municipal, avec une attention particulière pour les conceptions développées par les élus municipaux de Cracovie et de Varsovie. Elle suit particulièrement l'histoire de ces clivages régionaux au sein de l'Union des Villes Polonaises, qui se pose comme représentant des intérêts urbains dans le nouvel état. La centralisation imposée par ce dernier ira de pair avec le maintien de l'unité de l'union. 


\section{Le Municipalisme européen dans la première moitié du vingtième siècle: le réseau socialiste}

Cet article analyse la contribution du socialisme européen à la construction d'un réseau de réformateurs municipaux depuis la fin du igème siècle jusqu'aux années I950. Suite à l'échec de la tentative de constituer un réseau municipal socialiste au sein de la Deuxième Internationale, une fédération plus large de municipalités, l'Union Internationale des Villes/ International Union of Local Authorities (UIV/IULA) fut mise en place en I9I3. Les chefs socialistes belges, français, hollandais et anglais y furent particulièrement influents durant tout l'entre deux guerres, travaillant en coopération avec le mouvement coopérateur européen et le Bureau International du Travail de Genève. Cinquante années de débats et de projets lus à travers les Annales de la Régie Directe, périodique fondé par le socialiste français Edgard Milhaud, nous permettent de suivre le développement, l'essor et l'épuisement d'une génération de réformateurs municipaux, de leur contribution à la réflexion et à la pratique municipale jusqu'à leurs positions en faveur d'une Europe décentralisée, celle des villes et des autorités régionales.

\section{La Ville contre l'état ? Espoirs, rêves et limites du mouvement municipal international $1900-1960$}

Au début du vingtième siècle, la croissance des villes fur accompagnée par le développement d'un mouvement municipal international. L'Union Internationale des Villes/International Union of Local Authorities (UIV/IULA), créée en I9I3 à Gand, combinait l'approche politico-utopique et l'angle technico-professionnel. Sous le premier thème, on envisageait les municipalités urbaines comme les cellules de base d'une coopération à l'échelle européenne et mondiale, sous le deuxième la résolution des problèmes liés à l'urbanisation. Les deux approches partageaient une détermination identique à ignorer les limites administratives et les frontières nationales, et à rejeter la hiérarchie entre gouvernement local et gouvernement national. Entre les deux guerres, l'UIV/IULA s'éloigna graduellement de son inspiration utopiste, qui fut réinvestie par le Conseil des Communes d'Europe/Council of European Municipalities, créé à Genève en I95I, dans le but d'utiliser les municipalités comme bases pour promouvoir l'idée d'une Fédération Européenne des Etats.

\section{L'Europe par le bas: les jumelages de villes dans la France de la Guerre Froide}

Les jumelages urbains peuvent être considérés comme la première activité à impliquer les institutions municipales en tant que telles dans une action internationale de grande ampleur et de long terme. Le jumelage est un de ces 'traditions inventées' définies par Eric Hobsbawm. Les Fédéralistes français jouèrent un rôle décisif dans cette invention, qui regroupa un nombre important de municipalités européennes dans la perspective de construire l'union politique de la région. Très vite, des mouvements et définitions concurrentes se mirent en place, notamment avec le 'mouvement bilingue' qui s'empara des jumelages pour en faire l'outil d'une compréhension universelle qui aurait transcendé la division Est-Ouest. Une 'guerre des jumelages' fit rage pendant plusieurs années sur la scène française, guerre dont la compréhension appelle à l'inscription de ses enjeux dans le contexte de la Guerre Froide, et dont le déroulement participe de la dynamique de cette période. 


\section{Kurzfassungen}

\section{Auf Beziehungen setzen: ein munizipaler Beitrag}

Globalisierung, das Schlagwort des späten 20. Jahrhunderts, fordert auch den Historiker. Ein Weg zur Historisierung der sich hinter diesem Schlagwort verbergenden Phänomene ist der schon von anderen vorgeschlagene Blick auf die Beziehungen von Städten untereinander in einer Langzeitperspektive. Die Verbindungen zwischen städtischen Verwaltungen und die Debatten über dieses Thema sind von der historischen Forschung aus diversen Gründen vernachlässigt worden; sie können jedoch zur Geschichte der 'Konstruktion des Globalen' beitragen. Die Informationssysteme von Städteverbindungen - ihre Vektoren, Akteure, und Strukturen - definierten und förderten eine Reihe von möglichen universellen 'grenzüberschreitenden Formationen'. Diese Formationen beinhalteten sich wandelnde Kombinationen von Werten, kollektivem Handeln, Praktiken, Regeln, Organisationen und Individuen, die als möglicher Fortschritt für die Menschheit propagiert wurden. Durch die Analyse einiger dieser Diskurse über 'soziale Ordnung' oder 'Weltordnung' und die Art, wie sie sich mit munizipalen Verbindungen verknüpften, versucht dieser Artikel den munizipalen Beitrag zu leisten, der im Titel angekündigt ist.

\section{Die Wissenschaft, die nie existierte: 'Kommunale Wissenschaft' in Frankreich, 19I3-I949}

Die Versuche, eine 'munizipale Wissenschaft' zu entwickeln, bezeugen die Herausforderung, vor welche die Stadt die üblichen Verwaltungsmethoden in den ersten Jahrzehnten des 20. Jahrhunderts, speziell in Frankreich, stellte. Innerhalb Frankreichs und auch im internationalen Umfeld suchten Reformer nach neuen Wegen, um wissenschaftlich über Städte und deren Verwaltung nachzudenken. Dieser Artikel unternimmt eine Analyse einer 'munizipialen Wissenschaft', deren fragile Entwicklung nie vollständig in akademischen Kreisen akzeptiert wurde. Er untersucht die Versuche, einen wissenschaftlichen Rahmen zu finden für diejenigen Reformdiskurse, die von den Assoziationen städtischer Beamte entwickelt wurden und die durch Lehranstalten oder Zeitschriften, die sich der städtischen Verwaltung widmeten, weiterverbreitet wurden. Weiterhin wird der Beitrag skizziert, den dieser sowohl politische als auch wissenschaftliche Feldzug für die Erneuerung öffentlicher Aktivitäten durch lokale Autoritäten leistete.

\section{Die Union polnischer Städte während der Zweiten Polnischen Republik 1918-1939: Diskurse der Selbstverwaltung in einem geteilten Land}

Der neue polnische Staat wurde mehr als Ioo Jahre nach der Aufteilung Polens durch Preussen, Österreich und Russland gegründet. In vielerlei Weise wurden die geteilten polnischen Gebiete in die administrative Struktur der Besatzungsmächte einbezogen, und die Individuen, die in städtischen Fragen im neuen Staat aktiv waren, wurden in durch die Teilungsmächte errichteten Assoziationen sozialisiert. Polen wurde nicht nur ein Spielfeld für österreichische, preussische und russische Vorstellungen über lokales Regieren; es wies auch eine enorme Vielzahl städtischer Praktiken auf. Der Artikel analysiert die unterschiedlichen Bedeutungen von lokaler Regierung mit einem Schwerpunkt auf den von städtischen Verwaltungsbeamten aus Warschau und Krakau in der Union polnischer Städte verwendeten Begriffen. Innerhalb der Union gab es große Konflikte, aber die politische Entwicklung des polnischen Staates stärkte die Zentralisierung und so blieb die Union bestehen. 


\section{Europäischer Munizipalismus in der ersten Hälfte des 20. Jahrhunderts: das sozialistische Netzwerk}

Dieser Essay analysiert den Beitrag des europäischen Sozialismus zum Aufbau eines vielgestaltigen Netzwerks von Reformern auf dem Gebiet der Stadtpolitik seit dem Ende des I9. Jahrhunderts bis in die I950er Jahre. Da dieses Netzwerk innerhalb der Zweiten Internationale nicht funktionierte, wurde I9I 3 eine breitere internationale Verbindung von Städten, die IULA/UIV, vorgeschlagen. Belgische, französische, niederländische und englische sozialistische Führer hatten in der Zwischenkriegszeit den stärksten Einfluss innerhalb dieser Föderation und arbeiten eng mit kooperativen Bewegungen und dem International Labour Office in Genf zusammen. Die sich über 50 Jahre hinziehenden und durch die vom französischen Sozialisten Edgard Milhaud gegründete internationale Zeitschrift 'Les Annales de la régie directe' inspirierten Debatten erlauben es, die Entwicklung einer Generation lokaler Reformpolitiker vom Beginn munizipalistischen Denkens und Praxis bis hin zur Idee des Aufbaus einer dezentralisierten Europäischen Gemeinschaft von Städten und regionalen Autoritäten zu verfolgen.

\section{Städte gegen Staaten? Hoffnungen, Träume und Grenzen der europäischen munizipalen Bewegung I900-1960}

Das Wachstum der Städte zu Beginn des zwanzigsten Jahrhunderts wurde von der Geburt einer europäischen und internationalen munizipalen Bewegung begleitet. I9I3 wurde in Gent die Union Internationale des Villes/International Union of Local Authorities (UIV/ IULA) gegründet. Sie vereinte zwei Herangehensweisen, eine politisch-utopische, und eine technisch-professionelle, miteinander. Erstere konzentrierte sich darauf, Städte zu Initiatoren des Projekts einer weltweiten und europäischen Zusammenarbeit werden zu lassen; letzere beschäftigte sich vor allem mit den durch die Urbanisierung verursachten Problemen. Beide Ansätze vereinte die Entschlossenheit, ihre Ziele trotz kultureller und nationaler Barrieren sowie der administrativen Hierarchie zwischen lokaler und nationaler Regierung zu verfolgen. In der Zwischenkriegszeit verlor die UIV/IULA langsam ihren utopischen Impetus. Dieser wurde wieder aufgenommen durch das I95I in Genf gegründete Conseil des Communes d'Europe/Council of European Municipalities, welches zum Ziel hatte, Städte zum Ausgangspunkt einer Föderation der europäischen Staaten zu machen.

\section{Europe von unten: Städtepartnerschaften in Frankreich während des Kalten Krieges}

Städtepartnerschaften können als erster Versuch, städtische Institutionen in weiterreichende und dauerhafte internationale Aktivitäten einzubeziehen, gesehen werden. Sie entsprechen Eric Hobsbawms Definition einer 'invented tradition'. Die französischen Föderalisten spielten die entscheidende Rolle in diesem Prozess, der darauf abzielte, die europäischen Städte zusammenzuführen, um so die politische Union Europas zu fördern. Bald griff diese sogenannte bilingualistische Bewegung jedoch die Idee von Städtepartnerschaften als mögliches Mittel auf, um die weltweite Verständigung auch über die Ost-West Grenze hinweg voranzubringen. Dies führte jedoch $\mathrm{zu}$ einem heftigen Streit innerhalb der französischen Organisationen für die Städtepartnerschaften. Die Erfindung der Städtepartnerschaft muss deshalb zusammen mit den dynamischen politischen Spannungen innerhalb der französischen politischen Szene während des Kalten Kriegs studiert werden. 